\title{
A Practical Guide to Conducting Quality Improvement in the Health Care Setting
}

Eileen Murtagh Kurowski, MD, MS ${ }^{1,2,6,{ }^{*}}$

Amanda C. Schondelmeyer, MD, MS $S^{1,2,7}$

Courtney Brown, MD, MS ${ }^{1,2,4}$

Christopher E. Dandoy, MD, MS $1,2,3$

Samuel J. Hanke, $M D, M S^{1,2,8}$

Heather L. Tubbs Cooley, PhD, $R N^{2,5}$

\author{
Address \\ *,1Department of Pediatrics, Cincinnati Children's Hospital Medical Center, \\ Cincinnati, OH, USA \\ Email: Eileen.Murtagh-Kurowski@cchmc.org \\ 'James M. Anderson Center for Health Systems Excellence, Cincinnati Children's \\ Hospital Medical Center, Cincinnati, OH, USA \\ ${ }^{3}$ Division of Bone Marrow Transplantation and Immune Deficiency, Cincinnati \\ Children's Hospital Medical Center, Cincinnati, OH, USA \\ ${ }^{4}$ Division of General and Community Pediatrics, Cincinnati Children's Hospital \\ Medical Center, Cincinnati, OH, USA \\ ${ }^{5}$ Research in Patient Services, Division of Nursing, Cincinnati Children's Hospital \\ Medical Center, Cincinnati, $\mathrm{OH}$, USA \\ ${ }^{6}$ Division of Emergency Medicine, Cincinnati Children's Hospital Medical Center, \\ Cincinnati, $\mathrm{OH}$, USA \\ ${ }^{7}$ Division of Hospital Medicine, Cincinnati Children's Hospital Medical Center, \\ Cincinnati, OH, USA \\ ${ }^{8}$ Heart Institute, Cincinnati Children's Hospital Medical Center, Cincinnati, $\mathrm{OH}$, \\ USA
}

Published online: 17 October 2015

C) Springer International Publishing AG 2015

This article is part of the Topical Collection on Quality Improvement

Keywords Quality · Improvement • Variation • Plan-do-study-act (PDSA) cycle · Run chart · Implementation . Sustainability

\section{Opinion statement}

Quality improvement uses rigorous methodology to evaluate systemic changes to patient care processes in an effort to improve patient outcomes, the patient and family experience of care, and the safety and value of the care delivered. This article introduces the Model for Improvement, which was developed by the Associates for Process Improvement in the 
early 1990s using an adaptation of a real-life improvement project. The example will explore how a primary care practice uses the Model for Improvement to maximize the value and safety of care they deliver for children presenting with community-acquired pneumonia with an initial focus on appropriate first-line antibiotic treatment. The three fundamental questions which form the foundation of this approach are explored through the case example: (1) What are we trying to accomplish? (2) How will we know that a change is an improvement? (3) What changes can we make that will result in improvement? Examples of many of the fundamental tools used in the course of quality improvement work, such as a key driver diagram, run chart, and plan-do-study-act (PDSA) cycle, are explored throughout the text. Finally, a discussion of implementation and sustainability of improvement gains is introduced. This article serves as a primer on quality improvement in health care and serves as a foundation for subsequent articles in this issue as well as future learning.

\section{Introduction}

"Every system is perfectly designed to get the results it gets" (Batalden).

Over the last several decades, health care has become increasingly complex and costly $[1 \bullet \bullet]$. As a result, health care organizations struggle to provide safe, timely, and high-quality care, while still containing cost and satisfying patients and families. Modern quality improvement (QI), based on the theory and methods developed by Dr. Walter Shewhart and W. Edwards Deming in the 1920s, was originally applied in manufacturing industries in the mid-1900s. QI has been employed in the health care system since the days of Florence Nightingale in the 1850s [2] but gained renewed prominence after the publication of the Institute of Medicine reports in the late 1990s that highlighted challenges hospitals and health care workers face providing safe, high-quality care [3]. QI employs systematic changes to patient care processes that lead to improvement in patient outcomes and safety, the patient and family experience, and the value of care delivered.

While QI has become a widespread method for improving care, its acceptance as a rigorous scientific method has faced challenges. Traditional experimental research designs examine effects of one or two isolated interventions under controlled conditions. In contrast, QI methods involve multiple sequential changes over time and utilize continuous measurement and analysis. In complex and dynamic systems, QI allows for rapid testing and evaluation of new processes and methods for delivering care. QI science is rooted in quasiexperimental research design and strong statistical theory and, when systematically applied across sites, can produce generalizable knowledge about interventions that improve health care quality, safety, and value [4, 5]. In day-to-day practice, QI provides an essential set of tools specifically devised to address the gaps between the level at which a health care system currently functions and the level at which it could function. Practically speaking, and aside from its rigor as a scientific method and its ability to improve outcomes, leading and/or participating in QI has become a requirement for physicians to maintain their certification with many medical boards, including pediatrics [6].

While there are many methods by which the quality of patient care can be improved, including Lean and Six Sigma $[7,8]$, the Institute for Healthcare Improvement endorses a method based on the Model for Improvement, which was developed by the Associates for Process Improvement in the early 1990s. It focuses on five guiding principles [9]:

- Knowing why you need to improve

- Having feedback mechanisms to tell you if the improvement is happening

- Developing an effective change that will result in improvement

- Testing a change before attempting to implement

- Knowing when and how to make the change permanent

In this article, we will describe the practical application of the Model for Improvement for the health care setting organized around three fundamental questions [9]:

1. What are we trying to accomplish?

2. How will we know that a change is an improvement? 
3. What changes can we make that will result in improvement?

For clarity, we will use an example from the literature to emphasize the application of each step in the process to an actual problem in health care. The example [10•] outlined below is loosely based on previous work but has been altered to make the work applicable to a broader variety of practice settings:

You work at an outpatient pediatric practice with 9 other providers. As the physician leader in the clinic, you have been notified by the clinic's phone nurse about several complaints from parents whose children were prescribed antibiotics for community-acquired pneumonia (CAP) that were not covered by their insurance plans. Three families had been unable to afford to pay for the medicines out of pocket and were upset their child had to delay starting the antibiotic due to the issue. One family had to take their child to the Emergency Department (ED) for evaluation over the weekend after they were unable to fill the prescription. When you review the charts of children whose parents had called, you note substantial variability in antibiotics prescribed for CAP. One of your partners hears of the issue and mentions a recently published guideline for care of children with CAP. You decide to examine your practice group's current prescribing patterns for CAP compared with the guideline recommendations. Your chart review reveals that only $25 \%$ of patients in your practice receive first-line therapy that is in line with the new guideline. You present this data to your management who expresses support for work and appoints you to lead QI with the goal of improving this measure in the upcoming year.

\section{What are we trying to accomplish?}

\section{The aim statement}

The first task of a team seeking to make an improvement is to understand the "what." This is accomplished by the development of a specific aim statement. An effective aim statement is developed in collaboration with leadership and frontline staff in response to an observed problem. The aim statement may be broken up into a global aim, which denotes the long-term goals of the process under evaluation, and a specific aim, which identifies the more narrow scope of the current team's work. The specific aim for a project, sometimes referred to as a SMART aim, should be specific, measurable, actionable, relevant, and time-bound [9]. To do this, the specific aim statement should clearly state the process/system which will be the subject of the work, the desired outcome, the timeline during which the team will accomplish the work, and the magnitude of change that is expected. This will require identifying baseline and goal performance:

With this background, you draft the following global and specific aim statements for the project and plan to review with your team and leadership, once assembled:

Global Aim: Decrease unintended variation in the care of children with CAP in our pediatric practice to improve value, safety, outcomes, and patient and family experience of care.

Specific Aim: Increase proportion of children with CAP who receive appropriate first-line antibiotic treatment from a baseline level of $\mathrm{X}$ to goal level of Y by time Z.

Once the aim of the work has been defined, a team should be assembled which contains representation from all stakeholders in the process [11]. Stakeholders can include but are not limited to nurses, physicians, support staff, patients and family members, and hospital or clinic leadership depending on the process. These 
stakeholders must then work to map the workflow by creating a map from the beginning to the end of the care process the team is working to improve. From a high-level process map, the team can begin to identify where the potential failures in the current system have occurred, or could potentially occur in the future. These failures can be identified in a failure modes and effect analysis (FMEA), a systematic, proactive method of identifying causes of process failure and the risks associated with process failure in order to identify effective and targeted interventions [9, $12,13]$. A Pareto chart, or histogram representing the most common causes of process failure during the baseline data period, is another useful graphical method to help the team understand the most common causes of "failure." Using an FMEA and Pareto chart early on can focus interventions on the area with the greatest potential impact [9].

You assemble a team representing all key stakeholders in your group (physician, nurse practitioner, nurse, medical assistant, clerical staff, business director, facility manager) as well as a family member and review the aim statement. The team begins to work through the initial steps as follows:

- The current workflow is mapped by direct observation and all observations are shared among the team.

- The team then identifies potential failure modes for each step in the process, using a simplified FMEA (Fig. 1).


Fig. 1. Simplified failure modes and effects analysis (FMEA). 
- Once all potential failure modes have been identified, the team classifies each case where care did not adhere to the guideline.

- The areas in care that deviate from guidelines are classified as follows:

- Site of care delivery (e.g., ED instead of outpatient office care delivery)

- Clinical data collected at the visit

- Diagnostic testing performed at the visit

- Empiric therapy prescribed

- Office follow-up scheduled at an appropriate interval to ensure improvement with treatment regimen prescribed

- The team collects data on the process for 4 weeks and then constructs a Pareto chart (Fig. 2) to classify the reasons why patients did not receive appropriate first-line antibiotic treatment.

- Based on this information, the team proposes the following specific aim:

- Increase proportion of children with CAP who receive appropriate first-line antibiotic treatment from 25 to $90 \%$ within 6 months

Success of improvement work depends on a firm grounding in theory, which can be confusing for many health care practitioners looking to engage in or lead an improvement initiative. Davidoff et al. outline three levels of theory (grand, big, and small) to help clarify the role and importance of theory in improvement work [14•]. Grand theory is the most abstract and makes generalizations that apply across many domains. Big, or mid-range, theories bridge the gap between grand and small by outlining concepts that can be applied across improvement projects, such as the theory of diffusion of innovations [15]. Small or program theories are practical, accessible, and specific to a single improvement project or intervention. They specify, often in the form of a logic model or key driver diagram, the

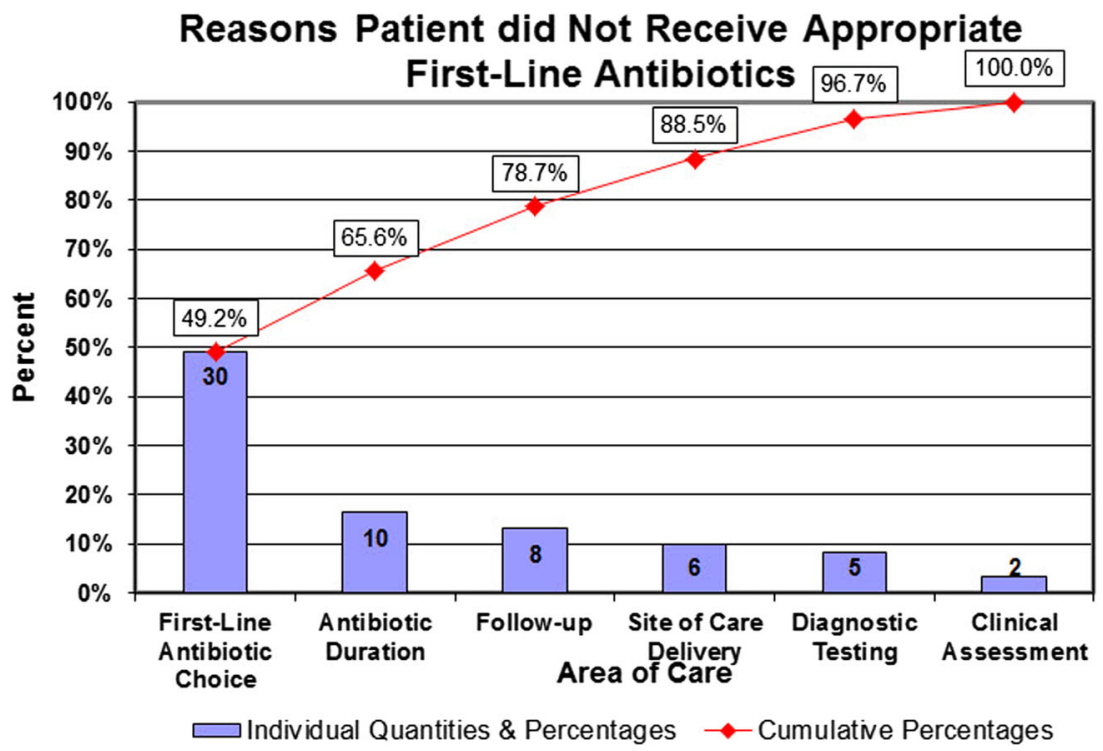

Fig. 2. Pareto chart of reasons for guideline non-adherence. 
components of an improvement project (or interventions) intended to address the intervention's expected outcomes (or drivers) leading to the desired improvement in the process (the specific aim) and the methods for assessing those outcomes.

It is important when generating the key driver diagram to get input from all stakeholders to ensure that all essential pieces of the process are identified. One method that can be used to describe the components of a key driver diagram is using the question "What?" to frame the drivers and "How?" to frame the interventions. The key driver diagram should be frequently revisited, and the program theory revised by the team as additional information is obtained during observation of the system and testing of interventions. It is likely that new interventions will be added or previous interventions modified from the iterative trial-and-learning process of the model for improvement. Once an initial list of key drivers has been agreed upon, it is time for the "good ideas" to be added to the key driver diagram. These good ideas are the proposed interventions based on your failure mode analysis outlined above. Arrows connecting the interventions to the appropriate key drivers can be used to denote which key driver(s) will be affected by a given intervention. These arrows will also be updated frequently, as the results of testing an intervention may reveal effects on a driver that had not previously been linked.

The team constructs a key driver diagram (Fig. 3) which includes the following components [9]:

- Global aim

- Specific aim

- Key drivers

- Interventions



Fig. 3. Key driver diagram. 


\section{How will we know that a change is an improvement?}

This second fundamental question in the Model for Improvement is answered with the observation of data and, more specifically, data over time. It is important for the team to identify appropriate measures to track with operational definitions of these measures that are clear and project-specific. There are three different types of measures which are often discussed in quality improvement work:

Outcome measure indicates the performance of the system under study and relates directly to the specific aim. This measure is often directly related to a patient or patient-care-related outcome.

- The team identifies "improved value of care for patient with CAP" as an outcome measure. The team struggles on how to measure value of care but believes that decreasing delays in antibiotic initiation, minimizing the number of changed prescriptions based on lack of insurance coverage, and minimizing the number of unplanned office and/or ED visits for the same illness would maximize the value of the care they deliver.

Process measure indicates if a key step in the process change has been accomplished.

- The team identifies "use of appropriate first-line antibiotics for CAP, as directed by the most recent evidence-based guideline" as a process measure. Given the difficulty in directly measuring the value of care, the team decides to start primarily tracking their process measure over time to assess the impact of their interventions.

Balancing measure indicates performance of related processes/outcomes to ensure that those measures are being maintained or improved, and also allows the team to monitor for unintended consequences of their process improvement work. Common examples in health care include adverse patient outcomes such as hospital readmissions, codes, or treatment failures.

- The team identifies multiple balancing measures:

- Number of parent/pharmacy calls about prescribed medication

- Number of unplanned return office visits and/or ED visits for the same illness

Ideally, a small, balanced family of measures, including at least one outcome measure, should be identified for an improvement initiative [16]. The team must work to understand how the data can be obtained for these measures, the accuracy of the data, and how often the data can be collected. Once the team has collected the data, it is important to understand the baseline performance of the system. Data for each measure is typically graphically plotted over time using run charts or Shewart (control) charts. The graphical nature of these charts makes them ideal for the evaluation of frequent changes in a measure since individual data points are displayed, allowing for maximum visualization of 
variation over time [17]. Understanding system variation is a critical concept when working to improve a process or outcome.

Run charts make it possible to determine if the variation in your system is secondary to changes made or to other inherent causes of variation in the system. Common cause (normal) variation is the variation that is inherent to the system. This variation is typically explained by unknown factors constantly active within the system. Common cause variation is often described as the "noise" in the system and, if singularly present, represents a "stable system." A stable system may be preferred if it is performing well; however, it may also represent a poorly performing system in which changes are needed.

Special cause variation is secondary to factors not inherent to the system. Special cause variation may be desired or not desired pending on the historical stability and performance of your system. It represents variation that is outside of the system's baseline experience. When a special cause event occurs, it is a signal that there is a new factor not typically part of the system impacting the system's performance. These events may represent favorable or unfavorable changes to the system. Ideally, during active improvement, special cause events signal improvements to the process or outcomes as a result of the team's interventions. Methods exist for identifying special cause based on the type of chart used to track the data over time. For run charts, there are probability-based rules to determine special cause, and control limits are calculated for Shewhart (control) charts as an additional method for determining special cause. There are many additional tools available which explain these rules and statistical calculations in greater detail and assist in appropriate statistical process control (SPC) interpretation [16]:

The team collects data using a standardized format for extraction from the medical record. They then use standard criteria to classify each therapy choice as a success (received the appropriate first-line antibiotic) or failure (did not receive the appropriate first-line antibiotic). This data is then plotted graphically over time as a run chart (Fig. 4).

\section{What changes can we make that will result in improvement?}

The third fundamental question in the Model for Improvement is answered through iterative testing of small changes to the process referred to as plan-dostudy-act (PDSA) cycles (also known as the Shewhart or Deming cycle) [9]:

The team utilized subject matter expertise and knowledge they had gained from mapping the process and identifying failure modes to identify potential interventions. The first intervention they focused on was:

- Error-proofing the process to order antibiotics for communityacquired pneumonia by integrating a decision-support tool at the point in the workflow where staff make the decision to prescribe

The PDSA cycle is a useful, four-step process to test theory and implement change. The key components of a PDSA cycle, with examples for each, are shown in Table $1[18,19]$. The four stages of the PDSA cycle are as follows: (plan) the change 


\section{Proportion of Children with Community-Acquired Pneumonia (CAP) Given Appropriate First-Line Antibiotic Treatment}

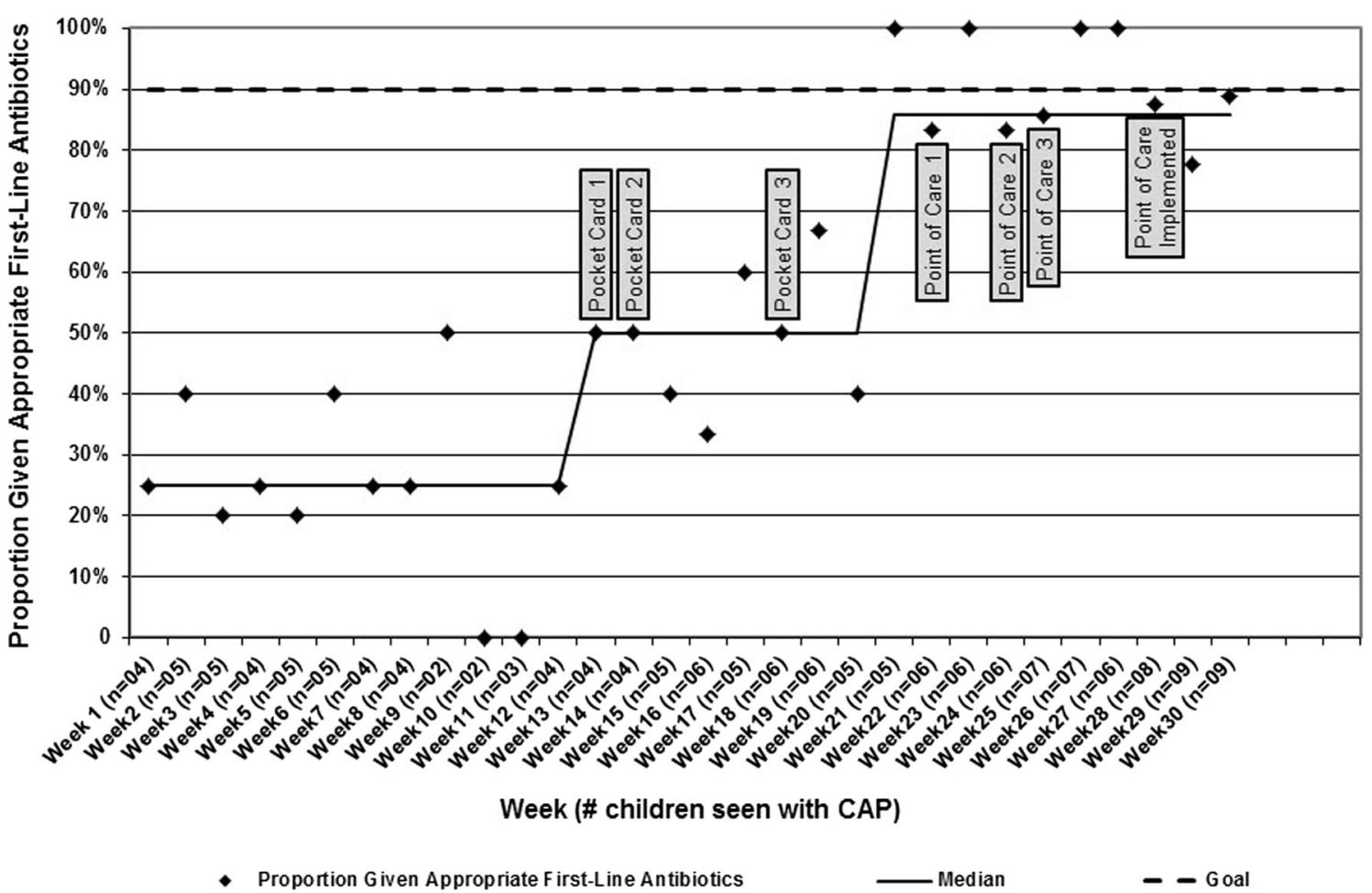

Fig. 4. Proportion of children with community-acquired pneumonia (CAP) given appropriate first-line antibiotic treatment.

to be tested or implemented, (do) carry out the test of change with careful measurement, (study) the data before and after the change and reflect on the learnings obtained, and (act) plan the next test. PDSA cycles are used to test an idea or theory through trial, assessing the change or impact, and making interventions based on these small tests. Each intervention is based on theory and should be tested on a small scale, sometimes on only one or two patients. Once the test shows improvement, these theories can be "ramped up" to include a larger population.

There are many benefits from starting small, and growing these tests to include large audiences. For example, when interventions are disruptive to opinions or existing processes, small tests can help generate buy-in from those involved in the testing to support larger-scale test. Multiple PDSA cycles are often linked together in a PDSA ramp, where small tests of changes are tested and adapted on progressively larger scale, to get from the initial idea to a change that is ready for implementation. Most projects will require multiple parallel PDSA ramps addressing multiple key drivers to achieve the aim. It is important to annotate all SPC charts with PDSA cycles/ramps to visually temporally track the impact of these cycles/ramps on the process, outcome, and balancing measures.

The team conducts multiple PDSA cycles that are linked together in a PDSA ramp, to investigate the best way to provide the recommendations for appropriate first-line antibiotic treatment at the point of care. The PDSA 
Table 1. Plan-do-study-act (PDSA) cycle

Plan:

- What is the objective of the test?

- What key driver(s) will the test impact?

- How will you measure the impact of the test?

-Note: This measure may be different from your measure for the overall project

- What do you predict will happen as a result of the intervention?

Do:

- Execute the test as planned

- Collect data, observations, and feedback from those involved in the test

- Record any circumstances that were not part of the initial test plan

Study:

- Was the test executed as planned?

What data was collected as part of the test? What was the effect of the intervention?

- Did the data collected match your prediction for what would happen?

Act:

- Take one of the following actions:

- Adapt - Improve upon the initial test idea based on results and continue with additional testing to further refine

- Adopt - Select changes for implementation. Plan for implementation and sustainability

- Abandon - Discard this change idea (based on results of testing) and try a new idea
Teams Plan for Testing:

- Trial a point of care tool to recommend appropriate first-line antibiotic therapy with 1 provider on 1 day

- Knowledge of appropriate treatment options at point of care

- Utility of the tool as rated by the provider and proportion of encounters in which tool produces an appropriate antibiotic recommendation

- The tool will be rated as very or somewhat useful by the provider and will be at least $99 \%$ accurate

Do:

- The test was executed with 1 provider for 1 day as planned

- The provider is surveyed on the utility of the tool after each visit and the recommendation made by the tool is compared to appropriate treatment to determine accuracy

- The trial version of the tool could only be run on a single computer

Study:

- Yes. 2 patients with community-acquired pneumonia were seen during the test

- The tool was rated as "somewhat useful" on both encounters and recommended the appropriate first-line antibiotic for both cases

- The data matched the team's prediction

Act:

- The team decides to adapt the tool based on feedback from the provider and observations of how the tool disrupted workflow. The team plans to repeat the test with another provider once the tool is adapted based on feedback

cycles linked together in this ramp are detailed below and annotated on the run chart in Fig. 4:

1. A simple pocket card given to a single provider when seeing a patient with CAP. The card reminds the provider of appropriate options for first-line antibiotic treatment. Provider finds the card helpful but is worried about not having access to it when needed.

2. The same pocket card is attached to all of the computers in the charting area of the clinic where providers often complete notes and write prescriptions. Two different providers each see one patient with CAP during the test. One provider found the card useful since he usually writes prescriptions in the charting area. The other provider wrote prescriptions in the patient room so did not see the cards in the charting area until later in the day. Both providers thought the information on the card would be beneficial to access at time of writing the prescription 
3. The same pocket card is given to all providers at the start of the clinic day to keep with them throughout all patient encounters. Three different providers each see one patient with CAP during the test. One provider misplaced their card and so could not reference it at the time of visit. The other two providers found the pocket card helpful but feared that they would lose it from one day to the next. All three providers thought that having access to the information on the card within the electronic medical record (EMR) where they write prescriptions would be more beneficial.

4. The similar PDSA ramp was conducted to test, initially with one provider on 1 day, the utility of a point of care tool, embedded within the EMR to recommend appropriate first-line antibiotics for CAP. This initial test was then ramped up to include all providers in the practice and is annotated on the run chart in Fig. 4.

\section{Implementation and sustainability}

Once the changes have been adapted to a point where the team identifies they are ready for adoption, the teams focus shifts to implementation of the change into everyday practice. This includes revising the process map to accurately depict the new process, revising any job or process descriptions to match the new process, and planning to train new members of the practice group on the new process.

The team revises the process map and identifies a new level of interaction with the electronic medical record is required for the adapted ordering process. This new interaction requires additional training for all providers who have ordering responsibilities. The materials used for orientation of new hires into the group practice are revised to reflect this new ordering process for all personnel. The team is excited about the success of their interventions and wants to ensure that these new processes are maintained when new and different problems arise in their practice. In order to sustain these efforts, the team develops a sustainability plan to ensure that the newly implemented processes and measures become hardwired into the daily clinic operations and individual clinician practices.

A sustainability plan includes the following:

- Deciding which performance measures will continue to be monitored using run charts?

- Developing a systematic (and ideally automated) process for obtaining and integrating the data that comprise the measures

- Determining who will be responsible for evaluating the performance measure on an ongoing basis (i.e., the process owner)

- Establishing measure parameters to guide the process owner's decisions about when to address deterioration in performance

- Articulating the process owner's role in addressing performance deterioration (e.g., power to reconvene the team and launch new series of explorations to understand why and how the process is failing) 
- Determining the frequency of re-evaluating measures to ensure consistency with current practice and to strategically determine when QI activities are no longer relevant or necessary for a given process (i.e., bimonthly and quarterly)

The team implements all of their successful interventions into everyday practice and works to develop a sustainability plan. They agree that the manager for the practice group will monitor the proportion of patients given appropriate first-line antibiotic therapy which is now generated as an automated report. If there is a special cause identified using SPC principles, the team leaders will investigate and determine if further intervention is needed, included reconvening the team.

\section{Conclusion}

The need to continually evaluate current performance and improve patient outcomes, safety, the patient and family experience, and the value of care delivered has become a fixture in health care. Understanding of and the ability to apply QI methods will help providers optimize care for their patients, meet requirements for certification, and be responsive to the need for cost containment in health care. This article provides an overview for health care providers on QI methods and in combination with others in this issue will lay the foundation for a deeper understanding of the multiple facets of and applications for health care improvement.

\section{Compliance with Ethics Guidelines}

\section{Conflict of Interest}

Eileen Murtagh Kurowski, Amanda C. Schondelmeyer, Courtney Brown, Christopher E. Dandoy, Samuel J. Hanke, and Heather L. Tubbs Cooley declare that they have no conflict of interest.

\section{Human and Animal Rights and Informed Consent}

This article does not contain any studies with human or animal subjects performed by any of the authors.

\section{References and Recommended Reading}

Papers of particular interest, published recently, have been highlighted as:

- Of importance

$\bullet \quad$ Of major importance

1.• Smith M, Saunders R, Stuckhardt L, McGinnis JM. Best care at lower cost: the path to continuously learning health care in America. National Academies Press; 2013. Institute of Medicine Report on the importance of continual learning and quality improvement in health care.

2. Chun J, Bafford AC. History and background of quality measurement. Clin Colon Rectal Surg. 2014;27(1):5-9.
3. Kohn LT, Corrigan JM, Donaldson MS. To ERR is human: building a safer health system. Washington: National Academies Press; 2000.

4. Ryan TP. Statistical methods for quality improvement. John Wiley \& Sons; 2011.

5. Strome TL. Healthcare analytics for quality and performance improvement. John Wiley \& Sons; 2013. 
6. The American Board of Pediatrics. Improving Professional Practice (Part 4). www.abp.org/content/ improving-professional-practice-part-4. Accessed September 14, 2015.

7. D'Andreamatteo A, Ianni L, Lega F, Sargiacomo M. Lean in healthcare: A comprehensive review. Health Policy. Feb 11 2015;11(15).

8. DelliFraine JL, Wang Z, McCaughey D, Langabeer 2nd JR, Erwin CO. The use of six sigma in health care management: are we using it to its full potential? Qual Manag Health Care. 2014;23(4):240-53.

9. Langley GJMR, Nolan KM, Nolan TW, Norman CL, Provost LP. The improvement guide: a practical approach to enhancing organizational performance. 2nd ed. San Francisco: Jossey-Bass; 2009.

10. Ambroggio L, Thomson J, Murtagh Kurowski E, et al. Quality improvement methods increase appropriate antibiotic prescribing for childhood pneumonia. Pediatrics. 2013;131(5):e1623-1631.

An exemplar paper on the use of quality improvement methods to improve clinical care.

11. Curtis JR, Cook DJ, Wall RJ, et al. Intensive care unit quality improvement: a "how-to" guide for the interdisciplinary team. Crit Care Med. 2006;34(1):211-8.

12. De Rosier J, Stalhandske E, Bagian JP, Nudell T. Using health care Failure Mode and Effect Analysis: the VA National Center for Patient Safety's prospective risk analysis system. Jt Comm J Qual Improv. 2002;28(5):248-67.

13. Israelski E, Muto W. Human Factors Risk Management for Medical Products. Handbook of Human Factors and Ergonomics in Health Care and Patient Safety, Second Edition: CRC Press; 2011:475-506.

14. Davidoff F, Dixon-Woods M, Leviton L, Michie S. Demystifying theory and its use in improvement. BMJ Qual Saf. 2015;24(3):228-38.

A recent manuscript on the importance of articulating theory prior to testing interventions.

15. Rogers E. Diffusion of Innovations. Fifth Edition ed. New York: Simon \& Schuster; 2003.

16. Provost LP, Murray S. The health care data guide: learning from data for improvement. John Wiley \& Sons; 2011

17. Perla RJ, Provost LP, Murray SK. The run chart: a simple analytical tool for learning from variation in healthcare processes. BMJ Qual Saf. 2011;20(1):46-51. doi:10. 1136/bmjqs.2009.037895.

18. Cleghorn GD, Headrick LA. The PDSA cycle at the core of learning in health professions education. Jt Comm J Qual Improv. 1996;22(3):206-12.

19. Speroff T, O'Connor GT. Study designs for PDSA quality improvement research. Qual Manag Health Care. 2004;13(1):17-32. 